\title{
Dependence of boron incorporation in delta layers on CVD diamond growth process and misorientation angle
}

\author{
M.A. Lobaev ${ }^{1}$, A.M. Gorbachev ${ }^{1}$, A.L. Vikharev ${ }^{1}$, D.B. Radishev ${ }^{1}$, V.A. Isaev ${ }^{1}$, \\ S.A. Bogdanov ${ }^{1}$, P.A. Yunin ${ }^{2}$, M.N. Drozdov ${ }^{2}$, J.E. Butler ${ }^{1}$ \\ ${ }^{1}$ Institute of Applied Physics of the Russian Academy of Sciences, Nizhny Novgorod, Russia \\ ${ }^{2}$ Institute for Physics of Microstructures of the Russian Academy of Sciences, Nizhny Novgorod, Russia
}

\section{Introduction}

Diamond is a promising material for the next generation of high-power and high-frequency semiconductor devices. In terms of its physical properties: high carrier saturation velocity $\left(2.7 \cdot 10^{7} \mathrm{~cm} / \mathrm{s}\right)$, high electron and hole mobility at low doping level, and record thermal conductivity, diamond significantly exceeds other semiconductor materials. Diamond is a wide-band gap semiconductor/insulator (band gap of about $5.5 \mathrm{eV}$ ), and partly because of this, all known dopants create the deep-lying levels with high activation energy. At room temperature only a very small fraction of the dopants are ionized. To create an acceptable level of conductivity, it is necessary to increase the level of doping, which inevitably leads to a decrease of the carrier mobility in diamond. To solve the problem of diamond doping the delta-layer technology is being developed. A nanometer thin layer of highly boron-doped diamond (the thickness of a few nanometers, $\mathrm{N}_{\mathrm{B}}>10^{20} \mathrm{~cm}^{-3}$ ) is introduced in the undoped highquality defect-free diamond. The achievement of high carrier mobility in this region requires the realization of sharp boundaries between doped and undoped material. This has been a rather difficult experimental problem. In this paper, we present the results of investigation of the boron incorporation into diamond delta doped layers as a function of the growth conditions and misorientation angle.

\section{Experiment}

Delta layers doped with boron were grown in homemade CVD reactor, described in detail in [1]. The reactor consists of a cylindrical cavity with a quartz tube placed on its axis. Inside the tube there is a substrate holder, over which a plasma is created using a magnetron at a frequency of $2.45 \mathrm{GHz}$. Inside the quartz tube, a laminar vortex-free gas flow is maintained at a flow of $900 \mathrm{sccm}$, which makes it possible to rapidly switch the composition of the gas mixture. Non-doped diamond is grown in a gas mixture of $\mathrm{H}_{2}+\mathrm{CH}_{4}\left(\mathrm{CH}_{4} / \mathrm{H}_{2}=0.1 \%\right)$. To grow the delta layer, this mixture rapidly (in comparison with the characteristic growth time of the diamond) changed to a gas mixture containing boron $\mathrm{H}_{2}+\mathrm{CH}_{4}+\mathrm{B}_{2} \mathrm{H}_{6} \quad\left(\mathrm{CH}_{4} / \mathrm{H}_{2}=\right.$ $\left.=0.1 \%, \mathrm{~B}_{2} \mathrm{H}_{6} / \mathrm{H}_{2}=0.1 \%\right)$ using a gas switch. The characteristic time for changing the composition of the gas mixture was found to be about 5 seconds. Typical epitaxial diamond growth rates were 40 to $80 \mathrm{~nm} / \mathrm{h}$. Due to presence of residual boron in the reactor the undoped diamond layers had low boron concentration, typically $10^{17} \mathrm{~cm}^{-3}$ or less, an amount which has little impact on the carrier mobility. Addition of hydrogen sulfide to gas mixture was used as a chemical getter to decrease the residual boron contamination during growth of undoped layers [2].

For the growth of CVD diamond delta layers doped with boron, IIa type high pressure high temperature (HPHT) grown diamond substrates from New Diamond Technologies with (100) orientation and size $3.0 \times 3.0 \times 0.5 \mathrm{~mm}$ were used. These substrates are nearly strain free as observed through crossed polarizers, with very low dislocation densities (ca. $10^{2} \mathrm{~cm}^{2}$ ). The substrates were mechanically polished to a surface roughness (Ra) of $0.1 \mathrm{~nm}$, measured with a Zygo NewView 7300 white light interferometer on an area of $0.22 \times 0.22 \mathrm{~mm}$. To remove defects introduced in the near surface region by polishing, a layer of 4-5 $\mu \mathrm{m}$ was homogeneously etched from the substrate in ICP plasma (Oxford Instruments, Plasmalab 80). As a result, defect-free substrates with an atomically smooth surface were used to grow the delta layers of the CVD diamond.

Measurement of the boron concentration in the grown samples was carried out by the secondary ion mass spectroscopy (SIMS) method using a time of flight SIMS setup (IONTOF TOF.SIMS-5). The depth of the etch craters for calculation of the analysis depth from the etching time was carried out using white light interference microscopes Talysurf CCI 2000 and Zygo NewView 7300. Sputtering was performed by $\mathrm{Cs}^{+}$ions with energy of $1 \mathrm{keV}$ at angle of $45^{\circ}$. The probing was carried out by $\mathrm{Bi}^{+}$ions with energy of $25 \mathrm{keV}$. The output of the cluster ion $\mathrm{C}_{2} \mathrm{~B}^{-}$was registered. Quantitative calibration of the mass spectrometer was performed using a test HPHT crystal implanted with boron ions with three different maximum concentrations $\left(10^{18}, 10^{19}\right.$ and $\left.10^{20} \mathrm{~cm}^{-3}\right)$.

The influence of growth conditions of CVD diamond on the incorporation of boron into the delta layer was investigated by growing structures containing several (35) delta layers. One of the growth process parameters was varied when growing each layer. Thus, the dependence of the boron incorporation on one of the growth parameters can be obtained within one growth process, which excludes the influence of such factors as the miscut angle and pre-treatment of the substrate, the "history" of the reactor, etc. In the case when several substrates were used to obtain the results, the substrates were selected with similar miscut angles of about $1^{\circ}$.

Figure 1 shows the dependence of the boron concentration in the delta layer on the substrate temperature with the methane content in the gas mixture $\mathrm{CH}_{4} / \mathrm{H}_{2}=0.07 \%$. During the experiment, all process parameters were kept constant, and only the temperature of the substrate was varied using an external heater. It is evident from Fig. 1 that boron is best incorporated into the delta layer in CVD diamond in the $850-900{ }^{\circ} \mathrm{C}$ temperature range. 
At higher temperatures, the efficiency of boron incorporation decreases.

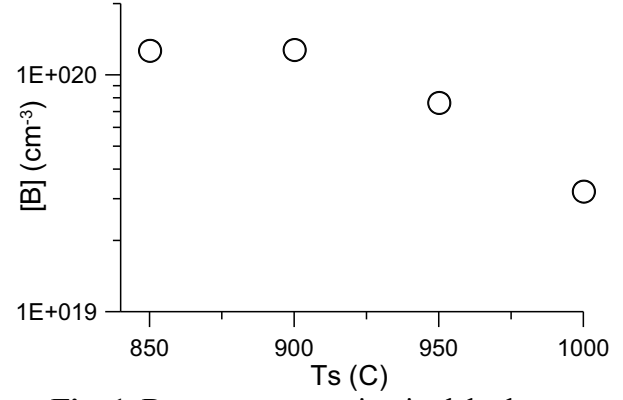

Fig. 1. Boron concentration in delta layer as function of substrate temperature

Fig. 2 shows the dependence of the boron incorporation into the delta layer on the $\mathrm{B} / \mathrm{C}$ ratio in the gas mixture at a methane content of $\mathrm{CH}_{4} / \mathrm{H}_{2}=0.07 \%$ (open circles) and $\mathrm{CH}_{4} / \mathrm{H}_{2}=0.14 \%$ (black circles). The temperature of the substrate was kept constant at $850{ }^{\circ} \mathrm{C}$. It is evident from Fig. 2 that with a $\mathrm{B} / \mathrm{C}$ ratio in a gas mixture of less than $20000 \mathrm{ppm}$, the incorporation of boron increases linearly with an increase of the $\mathrm{B} / \mathrm{C}$ ratio. At $\mathrm{B} / \mathrm{C}$ ratio in the gas mixture in the range from $20000 \mathrm{ppm}$ to $30000 \mathrm{ppm}$, saturation of the boron incorporation in the delta layer is observed. At a ratio values greater than $30000 \mathrm{ppm}$, the incorpo-ration of boron in the delta layer decreases. An increase in the methane content in the gas mixture from $\mathrm{CH}_{4} / \mathrm{H}_{2}=0.07 \%$ to $\mathrm{CH}_{4} / \mathrm{H}_{2}=0.14 \%$ leads to an increase in the incorporation of boron in the delta layer. Thus, to obtain heavily boron-doped diamond delta layers, it is necessary to select process parameters such that the $\mathrm{B} / \mathrm{C}$ ratio in the gas mixture does not exceed $20000 \mathrm{ppm}$.

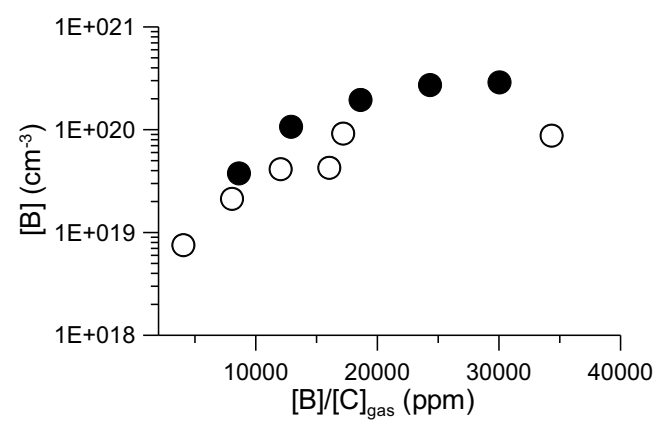

Fig. 2. Boron concentration in delta layer measured by SIMS as a function of $[\mathrm{B}] /[\mathrm{C}]$ gas ratio for two different methane content $\mathrm{CH}_{4} / \mathrm{H}_{2}=0.07 \%$ (open circles) and $\mathrm{CH}_{4} / \mathrm{H}_{2}=0.14 \%$ (black circles)

It is known that the boron incorporation into diamond during the CVD growth process depends on the miscut angle of the substrate [3]. To investigate the influence of the miscut angle on the incorporation of boron in the delta layer, the sample was used which surface is divided into three sectors with different angles of surface miscut angle relative to the crystallographic direction [001]. On that substrate, a structure containing 5 delta layers was grown with different $\mathrm{B} / \mathrm{C}$ ratios in the gas mixture. Fig. 3 shows the dependence of the boron concentration in the delta layer on the $\mathrm{B} / \mathrm{C}$ ratio in the gas mixture for different angles of deviation of the crystallographic direction [001] from the normal to the surface. The highest efficiency of boron incorporation in the delta layer is observed for the $0^{\circ}$ miscut angle. As can be seen from Fig. 3, the incorporation of boron in the delta layer saturates at $\mathrm{B} / \mathrm{C}$ ratio in the gas mixture over $20000 \mathrm{ppm}$ for all miscut angles. For miscut angles of $1^{\circ}$ and $3^{\circ}$, the incorporation of boron at a large $\mathrm{B} / \mathrm{C}$ ratio is almost equal.

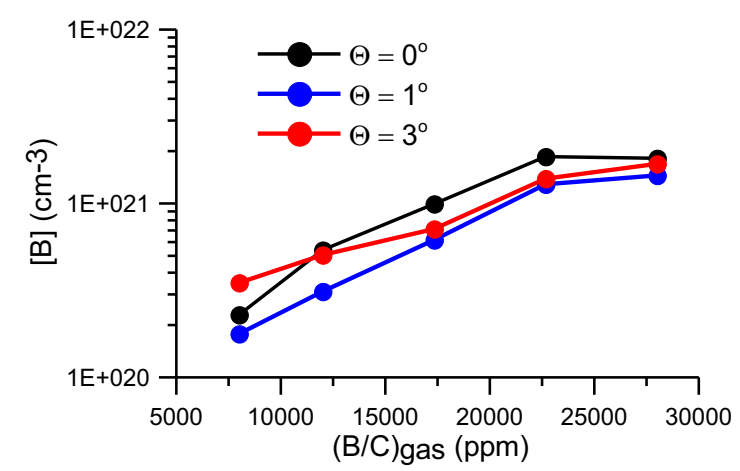

Fig. 3. Boron concentration in delta layer measured by SIMS as a function of $[\mathrm{B}] /[\mathrm{C}]$ gas ratio for different miscut angles

\section{Conclusion}

Noval MPACVD reactor was used for the investigation of boron incorporation in delta layers on CVD diamond growth process and misorientation angle. As the result of experiments the optimal diamond deposition parameters at which thin doped delta layers with boron concentration (5-10) $10^{20} \mathrm{~cm}^{-3}$ with thickness of 1-3 nm were found. This research allowed us to obtain heavily boron doped thin layers and to implement twodimensional hole "gas" in diamond with high mobility and hole concentrations.

\section{Acknowledgments}

This work was supported by Act 220 of the Russian Government (Agreement no. 14.B25.31.0021 with the host organization IAP RAS).

\section{References}

1. A.L. Vikharev, A.M. Gorbachev, M.A. Lobaev, A.B. Muchnikov et al. , Novel microwave plasma-assisted CVD reactor for diamond delta doping // Phys. Status Solidi (RRL), 2016, $10,324$.

2. S. C. Eaton, A. B. Anderson, J. C. Angus, Y. E. Evstefeeva et al. Co-doping of diamond with boron and sulfur // Electrochem. Solid-State Lett., 2002, 5, G65.

3. M. Ogura, H. Kato, T. Makino, H. Okushi et al., Misorientation-angle dependence of boron incorporation into (001)oriented chemical-vapor-deposited (CVD) diamond // Journal of Crystal Growth, 2011, 317, 60. 